\title{
Can the solar wind originate from a quiet Sun region?
}

\author{
J.-S. He ${ }^{1}$, C.-Y. Tu ${ }^{1,2}$, and E. Marsch ${ }^{2}$ \\ 1 Department of Geophysics, Peking University, Beijing, China \\ e-mail: chuanyitu@pku.edu.cn \\ 2 Max-Planck-Institut für Sonnensystemforschung, Katlenburg-Lindau, Germany \\ e-mail: marsch@mps.mpg.de
}

Received 21 September 2006 / Accepted 23 February 2007

\begin{abstract}
Context. It is well known that the fast solar wind originates from coronal holes (CHs). However, the question whether it can also originate from quiet Sun regions has not yet been answered.

Aims. To study this problem we analyze SOHO data obtained from observations made in a quiet Sun area. The data set includes far-ultraviolet data from SUMER, magnetic field data from MDI, and extreme-ultraviolet data from EIT.

Methods. We make a potential-field extrapolation of the coronal magnetic field and calculate the field lines from the photosphere up to $80 \mathrm{Mm}$ height. Those field lines which can be traced from the bottom to the top of the extrapolation box are called (locally) open field lines. By a combined analysis of the coronal magnetic field structures inferred from MDI data, the flows indicated by the Ne VIII Doppler shifts in the SUMER data, and the Fe XII radiance images from EIT, it is possible to study this problem in depth.

Results. We find that most of the sites with plasma outflow, which can be recognized by the Ne VIII blue shift, are not located in regions with an open magnetic field. Most likely, these outflows just correspond to plasma being delivered to magnetic loops. It is further found that, in a cross-section plane located at a height of $25 \mathrm{Mm}$, the pattern of open field lines intersecting that plane is consistent with the dark pattern of low radiance in the image of the Fe XII $19.5 \mathrm{~nm}$ line. Usually, small dark regions are considered to represent small $\mathrm{CHs}$, and thus are assumed to be sources of the solar wind. However, since here the source of the low emission appears to be located at a height of only $25 \mathrm{Mm}$, it seems more likely that this radiation originates near the foot points of large coronal loops.

Conclusions. Previous results obtained at middle latitudes on the quiet Sun indicated that sizable outflow velocities occur at the intersections of the network boundaries. This finding is also confirmed here. However, we could not identify most of these intersections as sources of the solar wind. Only a few small outflow regions might be sources. Yet, one dark area that we found on the EIT map seems to be connected with open field lines, and therefore it could be a source of the solar wind.
\end{abstract}

Key words. solar wind - Sun: corona - Sun: magnetic fields - Sun: transition region - Sun: photosphere

\section{Introduction}

Whether the solar wind can originate from the quiet Sun is an important question, which has been around for a long time but not yet been answered. Woo \& Habbal (2003) (and further references therein) found observational evidence being in favor of radial outflow from the quiet sun. It seems as if the solar wind during solar minimum can also originate from solar sources at middle and low latitudes, where the quiet Sun regions usually appear. Hassler et al. (1999) analyzed the Ne VIII blue shift in a quiet Sun region and found signatures of relatively large outflow velocity at the intersections of network boundaries at middle latitudes. It was further concluded that, since at middle latitudes the field lines are typically closed, the only possible location for an open field line is topologically at the intersection of the network boundaries. Outflow there was considered as a possible signature of a solar wind source. This finding was considered to be an essential step towards a better understanding of the sources of the solar wind in the transition region (TR) and lower corona. For a review of the TR see the book of Mariska (1992).

However, no magnetic field data were used in the cited work, and it was not checked if open field lines could indeed originate from quiet Sun regions at middle or low solar latitudes, and thus lead to outflow of solar wind there. Ohmi et al. (2003) found that open field lines can originate, and subsequently fan out widely, from the residual monopolar region in the vicinity of an active region, in which a low-speed stream with relatively abundant $\mathrm{He}^{2+}$ was formed. However, no detailed description of the solar source region of such a low-speed stream was given.

Recently, refined techniques for the extrapolation of the observed surface magnetic field to the corona were developed by Wiegelmann \& Neukirch (2002) who used and improved the method of Seehafer (1978) to extrapolate the magnetic field as measured in the photosphere, e.g. by MDI on SOHO. Using the force-free-field assumption, they routinely calculate the coronal magnetic field from a given photospheric magnetogram for a fixed value of the free model parameter $\alpha$. This extrapolation technique is usually applied to a finite rectangular segment of the solar atmosphere and provides the coronal vector magnetic field $\boldsymbol{B}(x, y, z)$, with Cartesian components $B_{x}, B_{y}$ and $B_{z}$, where $z$ is the vertical component, and $x$ and $y$ are the horizontal components of the position vector in the coordinate system.

This method was successfully applied by Marsch et al. (2004) to active regions, and also used by Wiegelmann et al. (2005) for calculating the fields in coronal holes (CHs). 


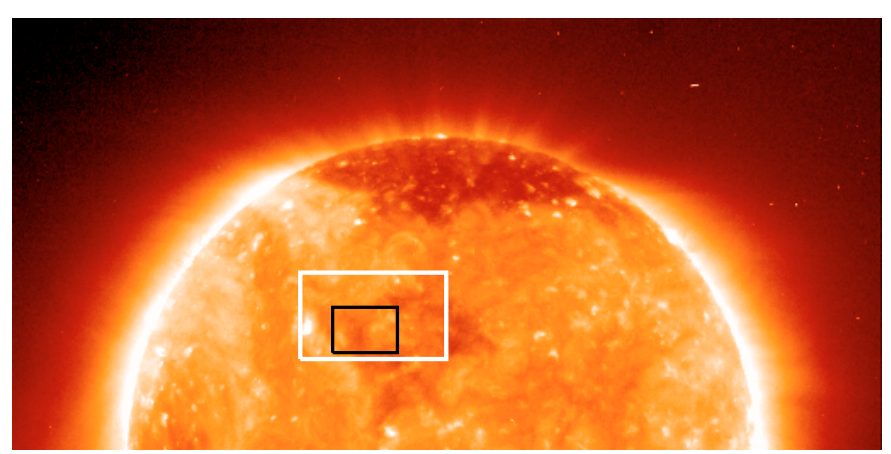

\section{EIT Fe $195 \AA$}

Fig. 1. EIT-image taken on 22 September 1996 at 01:05:56 UTC. The image has a pixel size of $2.629^{\prime \prime} \times 2.629^{\prime \prime}$. The white color rectangle superimposed on EIT-image shows the outline of the analyzed region, which is fixed at the MDI exposure time. The smaller black color rectangle surrounds the sub-region where a correlation analysis is made for the purpose of estimating the height of the weak Fe XII radiation.

Furthermore, Tu et al. $(2005 \mathrm{a})^{1}$ used this method to construct a magnetic funnel in a $\mathrm{CH}$ region. They found no principal differences in their results, when either making the assumption $\alpha=-1.1 \times 10^{8} \mathrm{~m}^{-1}$, which is the value derived for the force-free field in an AR by Marsch et al. (2004), or $\alpha=0$ for a simple potential field. Theoretically, steady open flux tubes should have no twist, which corresponds to $\alpha=0$, because twists will become Alfvén waves that can escape from the funnel.

Using this extrapolation technique, we will calculate the coronal magnetic field and with its help check if the sites where $\mathrm{Ne}$ VIII blue shifts are observed are connected with open magnetic field lines. We will analyze the SUMER data (for a detailed description, we refer to Wilhelm et al. 1995, 1997, 2004) to get the Ne VIII blue shift, the MDI data for calculating by a potentialfield extension the magnetic field, and an EIT $19.5 \mathrm{~nm}$ image for comparison with the open-field pattern. The purpose of the present data analysis is to establish in a quiet Sun region the spatial distribution of the open magnetic field lines, and then to study if the regions with Ne VIII blue shift and the dark regions on the EIT map are the sources of solar wind.

\section{Data analysis}

The quiet Sun region that we studied is shown in Fig. 1, where it is indicated by a white rectangle superposed on the background Fe XII $19.5 \mathrm{~nm}$ image that was taken on 22 September 1996 at 01:05:56 UTC. The SUMER data were obtained on 22 September 1996 at 00:40-08:15 UTC, and the MDI magnetic field data were taken at 03:15:30. The standard SUMER data-analysis procedures as described by Dammasch et al. (1999) and the methods for magnetic extrapolation as developed by Wiegelmann \& Neukirch (2002) were used for the

1 Here we point out a few minor corrections to be made in the papers by $\mathrm{Tu}$ et al. (2005a,b). In Tu et al. (2005a), (1) The MDI data used in that paper are from 01:36:30 on 21 September 1996. (2) In Fig. 3B, the unit of the $x$-axis should be changed from " $|B z| / G$ " to " $|B z| / 0.56 \mathrm{G}$ ". (3) In Table 1 , for both the Si II radiance and the C IV Doppler shift, the unit for magnetic field $B_{z}$ should be changed from " $\left|B_{z}\right| / 10 \mathrm{G}$ " to " $\left|B_{z}\right| / 5.6 \mathrm{G}$ ". These changes have no any impact on the conclusions and discussions of that paper. In Tu et al.(2005b), the MDI magnetic field data was observed at 03:15:30 UTC on 22 September 1996. We thank Mr. Jian-Sen He for pointing out these corrections. data analysis. The procedures used in this work are exactly the same as those already described in Tu et al. (2005b) and are therefore not addressed here.

The white rectangle superimposed on the EIT Fe XII image in Fig. 1 outlines the region corresponding to the used MDI magnetogram, ranging within the interval $\left[-442^{\prime \prime}, 9^{\prime \prime}\right]$ in the $x$-dimension and $\left[278^{\prime \prime}, 545^{\prime \prime}\right]$ in the $y$-dimension in solardisk coordinates. The position of this analyzed region, which is fixed at the MDI exposure time, changes since the Sun is rotating during the time difference between an EIT image exposure and an MDI magnetogram measurement, and during the time it takes SUMER to scan a raster image. For the sake of data comparison, the spatial pixels of the images made by SUMER and EIT were rotated from their position at the measurement time to the position at the time of the MDI exposure, under the assumption that the observed emissions did not change during the time it took to make these measurements. This assumption seems acceptable since we study phenomena related to the chromospheric network which have a typical life time of $20 \mathrm{~h}$.

The SUMER data we use here have before been presented and analyzed Hassler et al. (1999). They found strong blue shifts of the Ne VIII emission line at $77.0 \mathrm{~nm}$ at locations coinciding with intersections of the chromospheric network boundaries. The linear correlations between the line shift and the vertical components $\left|B_{z}\right|$ have been detected and studied by Tu et al. (2005b). The analysis of their data lead to the definition and evaluation of the correlation height, $h$, of the Ne VIII emission, yielding $h=4 \mathrm{Mm}$. In the present study we calculated the pattern of open field lines and compared it with the Ne VIII blue-shift pattern, and also with the dark patterns seen in the EIT image at $19.5 \mathrm{~nm}$.

Figure 2 shows the Fe XII radiance in the upper left subfigure and the magnetic $B_{z}$ component at the $z=0$ level in the upper right sub-figure, and at $z=10 \mathrm{Mm}$ and $z=25 \mathrm{Mm}$ in the bottom-left and bottom-right sub-figures, respectively. We can see the evolution with increasing height of the $B_{z}$ component. Apparently, the field forms structures over a wide range of scales. Dark-color contours of the Fe XII radiance are superimposed on the three $B_{z}$ images to emphasize possible spatial correlations. We thus can see that the low-intensity regions are located in the weak-field regions. We do not understand why this is so. Is a weak-field region a possible source of the solar wind in the quiet Sun? The situation is different from that in a $\mathrm{CH}$ in which the coronal funnels, which were established by Tu et al. (2005a) as sources of the solar wind, originate from strong photospheric field concentrations.

\section{Comparison of the open-field-line pattern with the blue-shift pattern of neon ions}

To identify the sources of the solar wind, we determined magnetic field lines reaching from the photosphere to the top plane of our box at a height of $z=80 \mathrm{Mm}$, thereby using the extrapolated magnetic field data. If a field line can be traced from $z=0 \mathrm{Mm}$ to $z=80 \mathrm{Mm}$ in our box, the field line is called locally open, or open field line to use a simple short term. If a field line does not go to the top plane it is called a closed field line. Many of the obviously closed field lines go back to the bottom plane of the box. Some other of the closed field lines intersect with the vertical boundaries of the box. Since our analysis is limited to the local 3-D box, we do not know if apparently open field lines do globally still belong to large coronal loops that reach beyond the size of our box, or whether they finally go out into interplanetary space. It is clear that only open field lines in the 3D box 

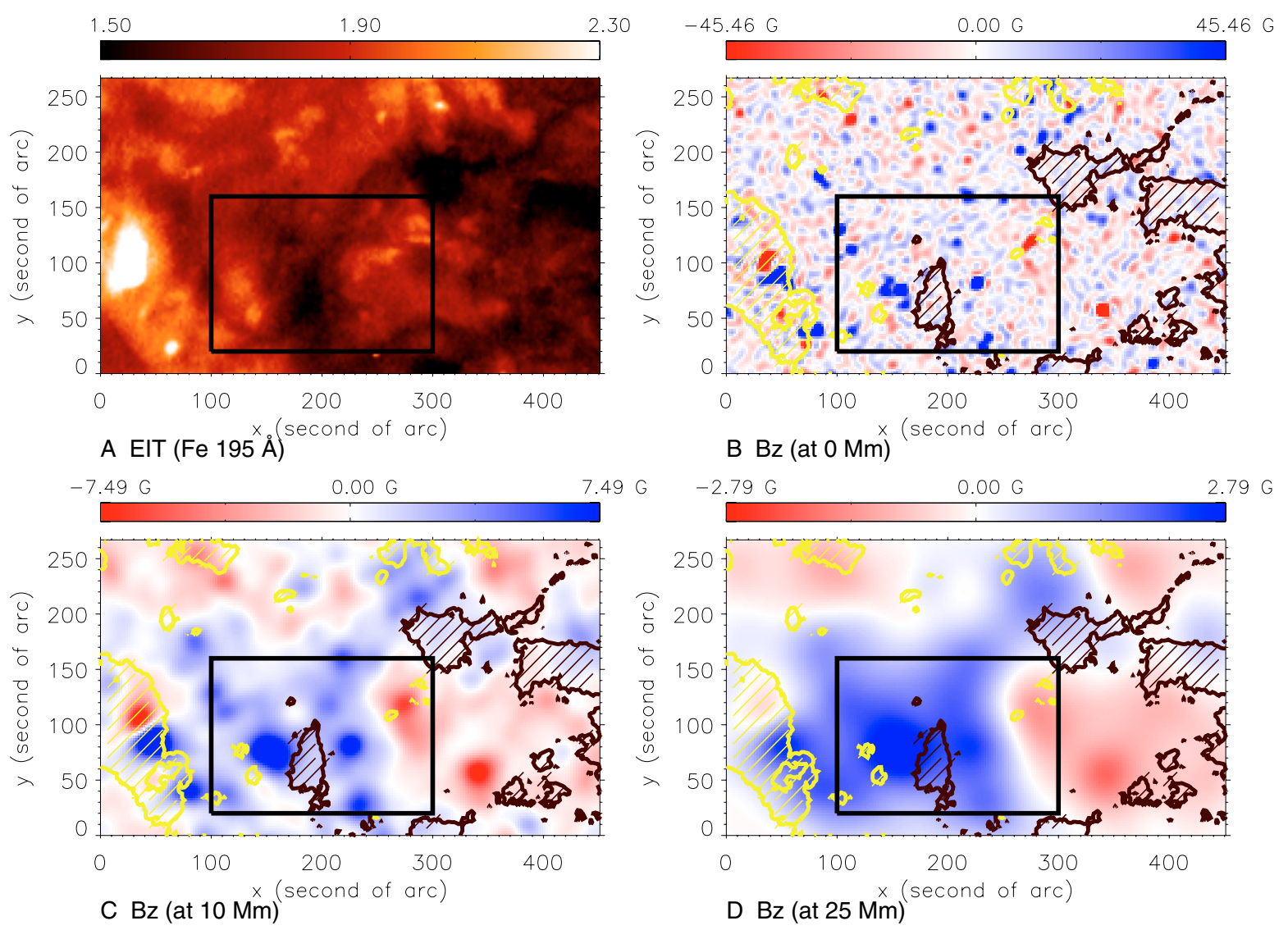

Fig. 2. Top left panel: the Fe XII $19.5 \mathrm{~nm}$ radiance in the region outlined by the white rectangle in Fig. 1. In all four panels of this figure the black color rectangles are placed at the same position as the small black rectangle in Fig. 1. The top right panel, bottom left panel and bottom right panel are maps of the $B_{z}$ distribution in the same region for a series of heights: $z=0 \mathrm{Mm}, 10 \mathrm{Mm}$ and $25 \mathrm{Mm}$. For the magnetic $B_{z}$ polarity, the blue color indicates an outward field direction, and the red color a direction towards the Sun. All of them are superimposed by two levels of slash-filled contours. The yellow ones cover the regions of strong Fe XII radiance $(\log ($ radiance $)>2.00)$, and the brown ones lay over the regions of weak Fe XII radiance $(\log ($ radiance $)<1.58)$.

can be considered as candidates for field lines really connecting the outer corona to the possible sources of the solar wind.

We now discuss if there are any open field lines that go through the intersections of the network boundaries, where the $\mathrm{Ne}$ VIII emission line reveals strong blue shifts. In Fig. 3 we provide a comparison between the sites with Ne VIII blue shifts and the intersection points (green crosses) of those field lines traced to the height of $25 \mathrm{Mm}$ in the extrapolation box with the crosssection plane of $z=4 \mathrm{Mm}$, which is the correlation height (for a definition see Tu et al. 2005b) for the Ne VIII emission line. The areas with strong $\mathrm{Ne}$ VIII blue shift are indicated by the closed dark-blue curves. The network-boundaries are reproduced from the paper by Hassler et al. (1999).

Since the height range over which the the field-line extrapolation is reliable can not unambiguously be determined, we present in Fig. 3 the results for field lines that are traced up to $25 \mathrm{Mm}$. From Fig. 3 we can see that some of the dark-blue contours are filled with green crosses (like at $y=70$ and $x=220$ ), but others are clearly not (like the closed contour at $y=170$ and $x=80$ ). Hence, these network intersections with strong blue shift seem generally not to be the sources of the solar wind. The corresponding outflows may just deliver plasma into small loops (with a height lower than $25 \mathrm{Mm}$ ), or into the lower corona and loops of the magnetic carpet.

Figure 4 shows the same comparison, but here the green crosses indicate the intersections of the field lines that are traced from the plane at $z=4 \mathrm{Mm}$ to the height of $z=80 \mathrm{Mm}$. Figure 4 confirms the finding that the strong Ne VIII blue shifts are located at some of the intersections of the network boundaries, where the magnetic field strength is shown to be high. However, we can clearly see that the five spots with the strongest blue shift are (at $x=80,100,275,350$, and 430) located well outside the intersection regions of open field lines. From this figure we can conclude that very few green crosses fall into the regions of strong blue shifts.

From Fig. 4 we can further see that some of the open-fieldline cross-section points tend to be collocated with some of the network lanes and the cross-sections of the network boundaries. This is consistent with the speculation made by Hassler et al. (1999) about the origin of the magnetic field lines in quiet Sun regions. However, no significant blue shifts are found in relation with these open field lines.

\section{Comparison of the open-field-line pattern with dark regions in EIT images}

In Fig. 5 we compare the dark regions in the image of the Fe XII $(19.5 \mathrm{~nm})$ line with the pattern of intersection points (indicated by green circles) of open field lines with the crossing plane at $z=25 \mathrm{Mm}$. We can see that these two patterns are similar and approximately coincide with each other for the middle part of the figure. The right-hand part of the image is also dark, but no open field line cross-section points are superposed on that region. This may result from boundary effects. The method we used for 


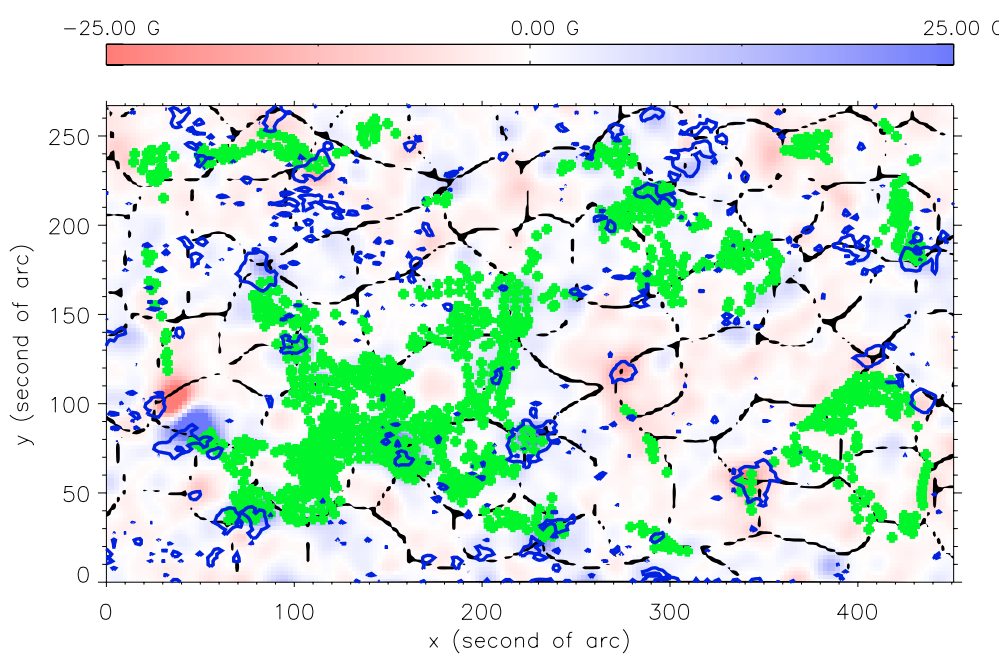

Fig. 3. Spatial relations between intersection points at $4 \mathrm{Mm}$ of open field lines, which are selected as such if they extend up to $25 \mathrm{Mm}$, and the strong Ne VIII blue-shift knots. The background image relates to the $B_{z}$ distribution at $4 \mathrm{Mm}$, with positive polarity characterized by light blue color and negative polarity by light red color. Broadly superimposed on it are the lanes of the chromospheric network in black color. The blue contours surround those regions where the speed inferred from the Ne VIII blue shift is larger than $5 \mathrm{~km} \mathrm{~s}^{-1}$. The dark-green plus signs show the intersection points of open field lines with the cross-section plane at a height of $z=4 \mathrm{Mm}$.

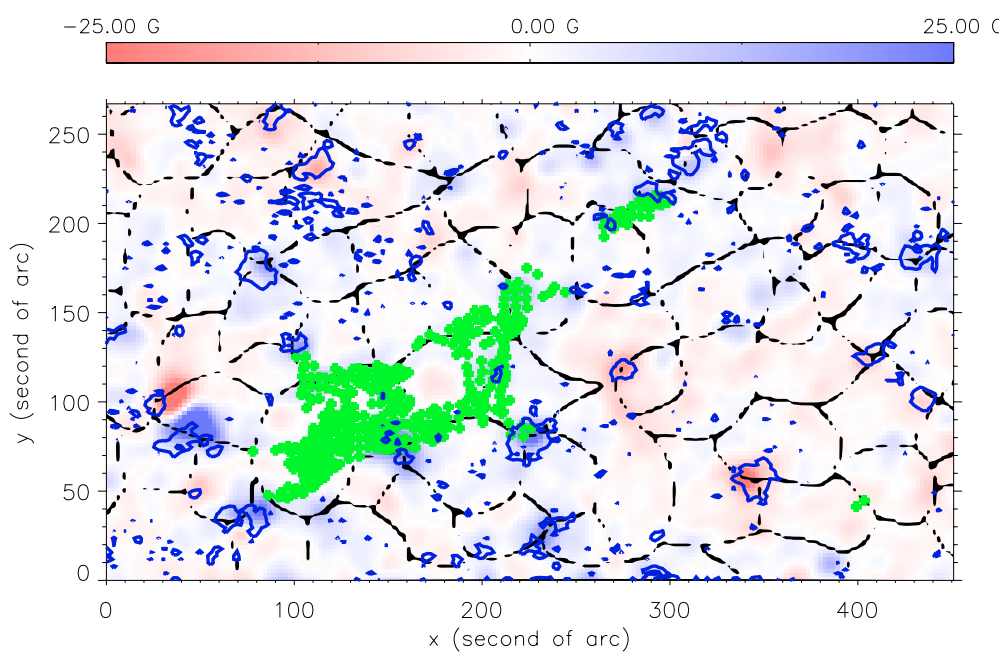

Fig. 4. Spatial relations between intersection points at $4 \mathrm{Mm}$ of open field lines, which are selected as such if they extend up to $80 \mathrm{Mm}$ rather than $25 \mathrm{Mm}$, and the strong Ne VIII blue-shift knots. The background image relates to the $B_{z}$ distribution at $4 \mathrm{Mm}$, with positive polarity characterized by light blue color and negative polarity by light red color. Broadly superimposed on it are the lanes of chromospheric network in black color. The blue contours surround those regions where the speed inferred from Ne VIII blue shift is larger than $5 \mathrm{~km} \mathrm{~s}^{-1}$. The dark-green plus signs show the intersection points of open field lines with the cross-section plane at a height of $z=4 \mathrm{Mm}$.

extrapolation may not always give reliable result for the magnetic field near the boundaries of the box.

The dark regions on the image of Fe XII (19.5 nm) just represent open-field-line magnetic structures. It is possible that these regions correspond to the source of the solar wind in the quiet Sun. Clearly, plasma outflow will cause a low density and hence small emissivity. Since no strong blue shifts are observed in these regions, we may argue that here continuous reconnection of middle-sized loops, as suggested by Tu et al. (2005a,c), may be required for generating the mass supply and initial (invisible) outflow of the tenuous nascent solar wind. It may also be that these open field lines are part of a large loop system, and that the plasma in the loops is rather static.

Figure 6 shows the distribution of 3-D magnetic field lines. The red color denotes the open field lines. The intersection plane showing the Fe XII intensity is placed at $z=25 \mathrm{Mm}$. We can see that the open field lines go through the dark region in the middle of the EIT image. This result suggests that the source region of the dark emission in Fe XII in the middle of the plot may be located at a height of about $25 \mathrm{Mm}$. If we define the open field lines as going to the top plane at $z=80 \mathrm{Mm}$, then we find the best fit of the pattern of open field lines with the dark region at $z=25 \mathrm{Mm}$. So the source of the dark region on the EIT image may be at heights above $25 \mathrm{Mm}$. We can also see that most of the magnetic field lines in the quiet Sun region are closed (with dark color). The outflow indicated by the neon blue shifts may correspond to a transport of plasma into these magnetic loops of various sizes.

From this figure we can also infer that the open field lines, whose intersection points largely coindice with the middle dark region, originate from several strong magnetic elements at $z=0$. Most of the magnetic flux tubes originating from these elements are closed. Only a small fraction of the magnetic flux is thus left 


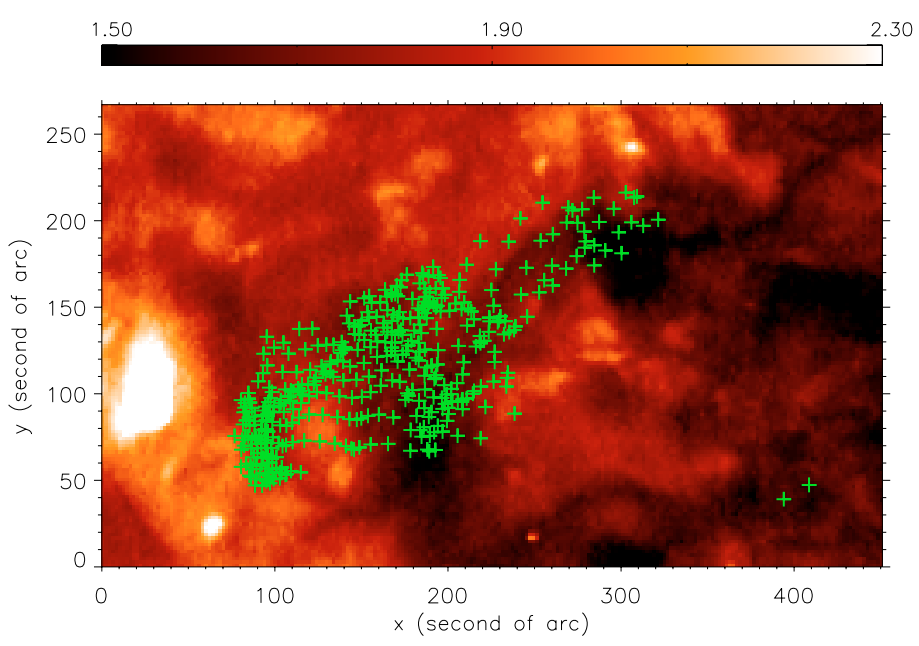

Fig. 5. EIT image of the Fe XII $(19.5 \mathrm{~nm})$ intensity superposed by the intersection points (indiated by green plus symbols) of the open magnetic field lines (that reach up to $80 \mathrm{Mm}$ ) with the intersection plane at the height of $z=25 \mathrm{Mm}$.

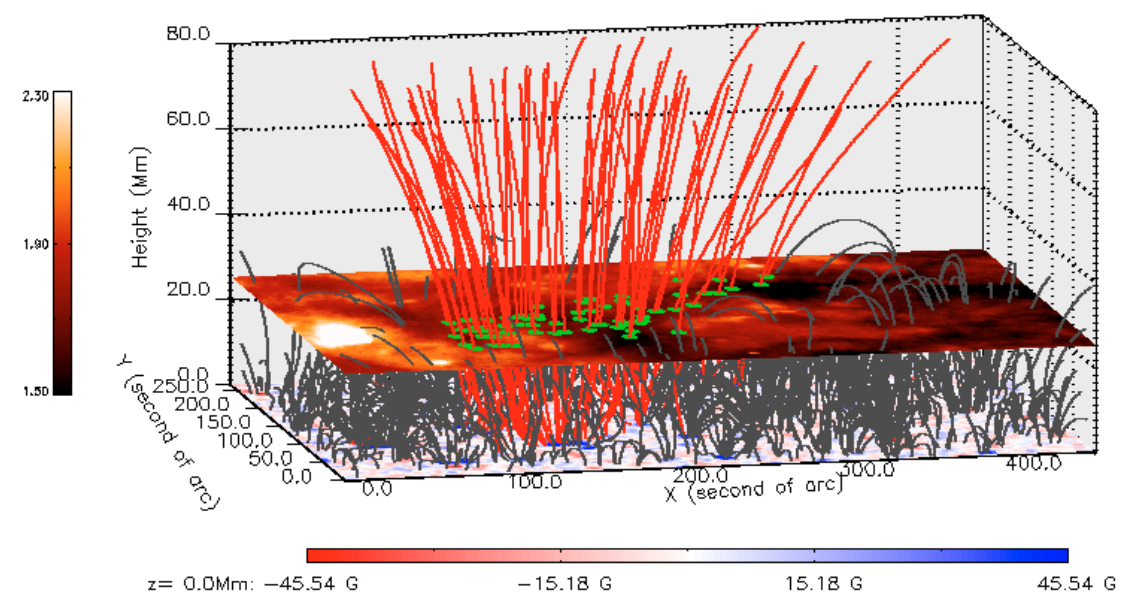

Fig. 6. Illustration of the 3-D magnetic field lines. The field lines are at $z=0 \mathrm{Mm}$ selected so that $\left|B_{z}\right|$ at their roots is larger than 7.5 gauss. The red color denotes open field lines and black closed ones. The Fe XII radiance image is placed at the height of $25 \mathrm{Mm}$. The intersection points of the open field lines with the plane at the height of $25 \mathrm{Mm}$ are indicated by dark-green dots.

to form this locally open flux region. That is why the magnetic field strength in this dark region is rather week.

In order to make our arguments more quantitative, we may use as a statistical measure a correlation analysis that corroborates the previous more qualitative comparison of the open magnetic flux distribution with the iron line radiance distribution, similar to what was done in previous work by Tu et al. $(2005 \mathrm{a}, \mathrm{b})$ who determined what they called the correlation height as a proxy for the estimated height of the line emission. The results of the statistical correlation are presented in the Fig. 7, which shows in the left panel the normalized correlation coefficient versus height in the corona in $\mathrm{Mm}$, and in the right panel the correlation plot between the line radiance and the ratio $\left|B_{z} / B\right|$ of the extrapolated magnetic field as an indicator for whether the field is more vertical or horizontal.

Clearly, the radiance is anti-correlated best at a height of $h=$ $25 \mathrm{Mm}$, which we call the correlation height that approximately defines where the emission is at a maximum. Furthermore, as discussed before, plasma on vertical open field lines shines less than plasma on horizontal (closed) field lines. The right panel shows the linear relation between the average of $\log$ (radiance) and $\left|B_{z} / B\right|$ divided in 10 bins ranging between 0.0 and 1.0. The mean values of $\log$ (radiance) in each bin are given by asterisks, with bars indicating one standard deviation. This plot confirms the correlation in the left panel.

\section{Conclusion and discussion}

Our data analysis confirms that the locations of strong blue shifts in Ne VIII coincide with the intersections of the chromospheric network boundaries, as it was found before by Hassler et al. (1999). However, most locations with strong blue shifts are not identified as the foot points of open field lines, and hence they may not be the source regions of the solar wind in the quiet Sun. The outflows being associated with strong blue-shifts may just represent the supply of plasma to the chromospheric magnetic carpet, i.e. to the many small closed-field loops in the solar TR and lower corona. Only a few regions of blue shift were found to be associated with field lines that reach out to $80 \mathrm{Mm}$. These field lines may be considered as open, and thus the corresponding regions with outflow may be identified as source regions of the solar wind. Although this result may be considered as partly supporting the conclusion by Hassler et al. (1999), it is statistically not robust, since the number of related data points is comparatively low. 

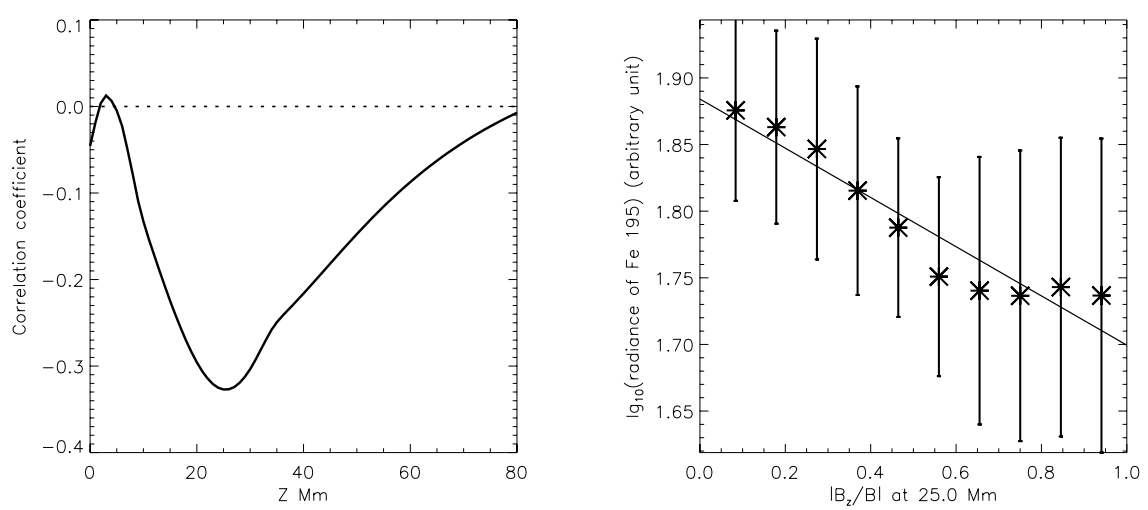

Fig. 7. Left panel: profile of the correlation coefficient (evaluated only in the sub-region indicated by the black box in Fig. 1) between $\left|B_{z} / B\right|$ and the Fe XII 195 radiance, as a function of height ranging from $0 \mathrm{Mm}$ to $80 \mathrm{Mm}$. At a height of $25 \mathrm{Mm},\left|B_{z} / B\right|$ is best inversely correlated with the logarithm of the radiance. The correlation coefficient at $25 \mathrm{Mm}$ is -0.33 , the absolute value of which is much larger than the critical value of the correlation coefficient, $R_{\mathrm{c}}=0.0154$, when the number of used pixels is 28000 . Thus the level of confidence is 0.99 . So the inverse correlation between $\left|B_{z} / B\right|$ and $\log$ (radiance) found at $25 \mathrm{Mm}$ is credible. Right panel: linear correlation analysis of the relation between $\left|B_{z} / B\right|$ and $\log$ (radiance) in the same sub-region and for the maximum correlation height of $25 \mathrm{Mm}$. The coefficient of the correlation between the 10 averaged points is -0.95 .

It should be pointed out that these conclusions were reached under the assumption of a potential filed extrapolation. Although Fig. 7 supports the use of such an extrapolation, this assumption may be questionable from the point of view that there is magnetic activity and that currents may flow in the corona. Therefore, the conclusion of Hassler et al. (1999) about the origin of the solar wind in the quiet Sun should be studied and checked again by considering the effects of magnetic field dynamics which a potential field can not describe.

The distribution pattern of the crossing points of the open field lines with the plane at $z=25 \mathrm{Mm}$ is roughly consistent with the dark region in the Fe XII $19.5 \mathrm{~nm}$ image of EIT. The dark patches in that image could correspond to sources of solar wind. But since no outflow, which is needed to supply plasma to an open magnetic flux tube from its bottom, is observed the original material for the solar wind must be provided by other means, for example by side flows or via reconnection with adjacent loops, just like in the scenario suggested by Tu et al. (2005a,c).

It is also possible that the open field lines are just small threads that belong to a larger coronal loop. The low density and low field strength expected inside such a thread will result in relatively weak ultraviolet emission.

Acknowledgements. The National Natural Science Foundation of China supported C.-Y. Tu and J.-S. He under contracts 40574078, 40336053 and 40436015. Tu and He are also supported by the Beijing Education
Project XK100010404. The SUMER project is financially supported by DLR, CNES, NASA, and the ESA PRODEX programme (Swiss contribution). SUMER, EIT, and MDI are instruments on board SOHO, an ESA and NASA mission. We thank the teams of MDI and EIT for the magnetic field data and the context images.

\section{References}

Dammasch, I. E., Wilhelm, K., Curdt, W., \& Hassler, D. M. 1999, A\&A, 346, 285

Hassler, D. M., Dammasch, I. E., Lemaire, P., et al. 1999, Science, 283, 810

Ohmi, T., Kojima, M., Hayashi, K., et al. 2003, in Solar Wind Ten, Proceedings of the Tenth International Solar Wind Conference, ed. M. Velli, R. Bruno, \& F. Malara, Pisa, Italy, 17-21 June 2002, 137

Mariska, J. T. 1992, The Solar Transition Region (Cambridge: Cambridge Univ. Press)

Marsch, E., Wiegelmann, T., \& Xia, L. D. 2004, A\&A, 428, 629

Seehafer, N. 1978, Sol. Phys., 58, 215

Tu, C.-Y., Zhou, C., Marsch, E., et al. 2005a, Science, 308, 519

Tu, C.-Y., Zhou, C., Marsch, E., et al. 2005b, ApJ, 624, L133

Tu, C.-Y., Zhou, C., Marsch, E., et al. 2005c, Proceedings of SW 11-SOHO 16, Connecting Sun and Heliosphere, Whistler, Canada, 12-17 June 2005, ESA SP-592

Wiegelmann, T., \& Neukirch, T. 2002, Sol. Phys., 208, 233

Wiegelmann, T., Xia, L. D., \& Marsch, E. 2005, A\&A, 432, L1

Wilhelm, K., Curdt, W., Marsch, E., et al. 1995, Sol. Phys., 162, 189

Wilhelm, K., Lemaire, P., Curdt, W., et al. 1997, Sol. Phys., 170, 75

Wilhelm, K., Dwivedi, B. N., Marsch, E., \& Feldman, U. 2004, Space. Sci. Rev., 111 , Issue 3, 415

Woo, R., \& Habbal, S. R. 2003, in Solar Wind Ten, Proceedings of the Tenth International Solar Wind Conference, ed. M. Velli, R. Bruno, \& F. Malara, Pisa, Italy, 17-21 June 2002, 55 